\author{
SERIES "RECENT DEVELOPMENTS IN PULMONARY INFECTIONS" \\ Edited by M. Woodhead and T. Schaberg \\ Number 2 in this Series
}

\title{
Severity assessment in community-acquired pneumonia
}

\author{
S. Ewig*, H. Schäfer*, A. Torres ${ }^{+}$
}

Severity assessment in community-acquired pneumonia. S. Ewig, H. Schäfer, A. Torres. (C) ERS Journals Ltd 2000.

ABSTRACT: In current guidelines for the management of adults with communityacquired pneumonia (CAP), the triaging decision about hospitalization or intensive care unit (ICU) admission, and, as a consequence, selection of initial antimicrobial treatment is largely based on the assessment of pneumonia severity.

The proposed severity criteria are mainly derived from studies determining predictors of adverse outcome. These include age, male sex, comorbidity, acute respiratory failure, severe sepsis and septic shock, extension of radiographic infiltrates, bacteraemia and CAP through several different pathogens such as Streptococcus pneumoniae, Staphylococcus aureus, Gram-negative enteric bacilli (GNEB), and signs of disease progression within the first $48-72 \mathrm{~h}$. In addition, prediction rules and need for a complicated course in ambulatory and hospitalized patients, for the individual risk of death have been developed which may be helpful in determining the patient who might require hospitalization or intensive care, respectively.

Risk classifications such as the scores developed by FINE et al. [40] are not only useful for identifying low risk patients who might safely be treated as outpatients, but apparently they will also play a major role in the evaluation of processes and outcomes of care for patients with CAP. Recent investigations have provided objective criteria for the definition of severe CAP requiring ICU admission. Whether the detection of infiltrates in the chest radiographs of patients with acute lower respiratory tract infection (LRTI) suggestive of mild pneumonia has an independent prognostic impact which fundamentally affects the concept of mild LRTI remains to be seen.

Based on objective criteria for severity assessment it will be possible to define interventions aimed at reducing hospital admission rates, define a risk-adapted antimicrobial treatment regimen, reduce costs for antimicrobial treatment and supportive measures, shorten hospital stay, and, thereby, improve the quality of care for patients with community-acquired pneumonia.

Eur Respir J 2000; 16: 1193-1201.
*Dept of Medicine II, Cardiology and Pneumology, Bonn, Germany. 'Hospital Clinic i Provincial, Universitat de Barcelona, Barcelona, Spain.

Correspondence: S. Ewig

Medizinische Universitätsklinik und Poliklinik II

Innere Medizin/Kardiologie und Pneumologie

Sigmund Freud Straße 25

53105 Bonn

Germany

Fax: 492282879017

Keywords: Outcome

pneumonia

prognosis

risk factors

severity

Received: December 311999

Accepted after revision July 272000
Current guidelines for the treatment of patients with community-acquired pneumonia (CAP) are based on estimations of pneumonia severity which allow a riskadapted approach to the decision about the treatment setting and the selection of initial empiric antimicrobial regimen [1-4]. The framework of severity assessment includes outpatient, hospitalized, and intensive care unit (ICU) pneumonia which corresponds to mild, moderate, and severe pneumonia. In particular, the guidelines of the American Thoracic Society (ATS) [1], the European Respiratory Society (ERS) [2, 3], and of the Infectious Disease Society of America (IDSA) [4] all agree in that severe CAP represents a pneumonia syndrome of its own which requires a distinct approach to diagnosis and treatment. This concept is also based on increasing evidence that severe CAP is more frequently associated with bacteraemia and distinct micro-organisms such as Legionella spp., Gram-negative enteric bacilli (GNEB), and Pseudomonas aeruginosa [5, 6].
Thus, the assessment of severity has become one of the most important issues in the management of patients presenting with CAP. In the following, the authors review the evidence from the recent literature with regards to prognostic factors, prognostic rules and severity criteria and their implications for clinical decision making. At the same time, emphasis will also be placed on explaining unresolved critical issues with respect to severity assessment.

\section{Detecting mild pneumonia}

Pneumonia is usually considered to represent a more serious illness than nonpneumonic acute lower respiratory tract illness such as acute bronchitis generally requiring antimicrobial treatment. If this holds true for mild pneumonia also, then the first step of severity assessment of a patient with symptoms of lower respiratory tract infection (LRTI) would be identical to the diagnostic 
evaluation for the presence of pneumonia. Otherwise, it would be more practical to restrict the diagnostic work-up of such patients to the assessment of severity of LRTI regardless of the potential presence of pneumonia in order to decide on which patient would require empiric antimicrobial treatment at all.

The ERS guidelines are based on the recognition that it may be very difficult in clinical practice to differentiate bronchitis and mild pneumonia. Therefore, an effort to differentiate acute bronchitis from pneumonia by a chest radiograph is only recommended in patients presenting with focal chest signs, the single clinical sign with the best predictive potential for the presence of pneumonia [7]. In fact, LRTI represents a spectrum ranging from mild bronchial to overwhelming parenchymal infection. Single clinical signs as well as diagnostic rules consisting of a set of criteria do not provide satisfactory performances in predicting the presence of pneumonia [8-11]. Chest radiographs are also only of limited value in the imaging of mild infiltrates $[12,13]$. Moreover, since only every 10th or 20th patient with LRTI is expected to have true pneumonia [14], thus resulting in a low prevalence, the positive predictive value of any diagnostic rule will be low. Thus, a sharp distinction will not be possible in every case of mild LRTI. On the other hand, diagnostic rules have been shown to achieve acceptable negative predictive values [15]. Therefore, in the absence of clinical signs such as fever, tachypnoea, tachycardia, cough, expectoration and rales, pneumonia is improbable. Otherwise, the presence or absence of pneumonia can only be determined more reliably by a chest radiograph.

Up to the present day, however, no study has assessed whether the presence of infiltrates visible in the chest radiographs of patients with symptoms of mild LRTI has any implications in terms of outcome as compared to patients without infiltrates but similar clinical symptoms. This would be a pragmatic and clinically meaningful way to obviate the somewhat academic distinction between acute bronchitis and mild pneumonia. Moreover, no study has investigated whether a strategy which generally requires a chest radiograph to confirm pneumonia in patients with equivocal clinical features is superior to a strategy which is restricted to a clinical decision about the indication for empiric antimicrobial treatment. Evidently, both questions are of eminent importance in order to define an adequate strategy of diagnostic work-up and severity assessment in patients presenting with symptoms compatible with mild LRTI.

\section{Risk factors for a complicated course of CAP}

Around $80 \%$ of LRTI thought to represent true pneumonia are mild and can be treated on an outpatient basis [16]. However, in patients without obvious reasons for immediate hospitalization, the triaging of outpatients for hospital admission may be difficult. Therefore, a scoring system was derived from a retrospective chart review and validated prospectively [17]. In the derivation protocol, patients with a predefined disease complication present during the initial evaluation which required immediate hospitalization were excluded. The remaining patients were classified as hospitalization necessary or unnecessary. Independent predictors for the need for hospitalization and their relative weights were: 1) serious comorbid illness (3 points), 2) pre-existing lung disease (2), 3) multilobar infiltrates (2), 4) observed or likely aspiration (2), and 5) symptom duration $<7$ days or $>28$ days (1). Patients with low risk scores of $0-2$ points rarely had complications $(2 \%$ in both derivation and validation population) whereas patients with high scores $\geq 6$ points had frequent complications $(69 \%$ in derivation and $67 \%$ in validation population). Patients with intermediate scores $3-5$ had complications in $19 \%$ and $25 \%$, respectively. These data impressively show that simple clinical and radiological data can be helpful in the severity and risk assessment of ambulatory patients.

A limited but probably important proportion of patients initially judged to have mild pneumonia require hospitalization during the course of the disease because of the failure to respond to the initial empirical antimicrobial treatment or even due to clinical deterioration. In one study, five variables were found to predict treatment failure of patients initially assigned to ambulatory treatment [18].

Table 1. - Risk factors for a complicated course of community-acquired pneumonia (including death) according to the American Thoracic Society guidelines for the management of patients with community-acquired pneumonia

Premorbid epidemiological and historical factors

Age $>65$ yrs

Suspicion of aspiration (gastric or oropharyngeal secretions)

Congestive heart failure

COPD, bronchiectasis

Diabetes mellitus

Chronic alcohol abuse

Malnutrition

Chronic renal failure

Chronic liver disease of any aetiology

Hospitalization during the prior 12 months

Previous splenectomy

Altered mental status

Physical findings

Temperature $>38.3^{\circ} \mathrm{C}$

Respiratory rate $\geq 30 \cdot \mathrm{min}^{-1}$

Systolic blood pressure $<90 \mathrm{mmHg}$, diastolic blood pressure $<60 \mathrm{mmHg}$

Extrapulmonary involvement (septic arthritis, meningitis, etc.)

Mental confusion or decreased level of consciousness

Laboratory findings

Leukocytosis $>30 \times 10^{9} \cdot \mathrm{L}^{-1}$ or leukopenia $<4 \times 10^{9} \cdot \mathrm{L}^{-1}$

Hematocrit $<30 \%$ or haemoglobin $<9 \mathrm{~g} \cdot \mathrm{dL}^{-1}$

$\mathrm{Pa}_{\mathrm{a}, \mathrm{O}_{2}}<60 \mathrm{mmHg}$ or $\mathrm{Pa}_{\mathrm{a}} \mathrm{CO}_{2}>50 \mathrm{mmHg}$ at $\mathrm{FI}, \mathrm{O}_{2} 0.21$

Blood urea nitrogen $\geq 20 \mathrm{mg} \cdot \mathrm{dL}^{-1}$ or creatinine $\geq 1.2$ $\mathrm{mg} \cdot \mathrm{dL}^{-1}$

Radiographic findings

$>1$ lobe involvement

Presence of cavity

Rapid radiographic spread

Presence of pleural effusion

Others

Requirement for mechanical ventilation

Evidence for sepsis or organ dysfunction (metabolic acidosis, increased prothrombin or partial thromboplastin time, decreased platelets, presence of fibrin split products)

COPD: chronic obstructive pulmonary disease; $\mathrm{Pa}_{\mathrm{a}} \mathrm{O}_{2}$ : oxygen tension in arterial blood; $\mathrm{Pa}_{\mathrm{a}} \mathrm{CO}_{2}$ : carbon dioxide tension in arterial blood; $\mathrm{FI}, \mathrm{O}_{2}$ : inspiratory oxygen fraction. Adapted from [1]. 
These included: 1) age $>65 \mathrm{yrs} ; 2)$ the presence of comorbid illness; 3 ) fever $\left(>38.3^{\circ} \mathrm{C}\right)$; 4$)$ immunosuppression; and 5) the presence of high-risk aetiologies (including S. aureus, GNEB, aspiration, and postobstructive pneumonia). The risk of treatment failure increased linearly with the number of risk factors present. However, the results of this study are hampered by two important issues: 1) in view of different susceptibility to opportunistic pathogens, patients with severe immunosuppression should not be classified as having CAP; instead, these patients require a different approach to diagnosis and treatment [19]; 2) the aetiology is not known at the initial clinical evaluation of the patient and, therefore, can not form part of the initial risk assessment. Nevertheless, the crucial role of age and comorbidity remains evident.

The ATS guidelines have outlined a variety of factors that increase the risk for a complicated course for CAP (including death) and recommended that hospitalization should be strongly considered when multiple risk factors are present [1]. These factors are listed in table 1. The selection of risk factors was based on prognostic studies and clinical expertise. In fact, in a multicentre retrospective analysis of outcomes in patients with CAP, a linear correlation was observed between the number of risk factors according to these ATS guidelines and a variety of outcome measures, including mortality, admission at the ICU, mean hospitalization time, and mean cost [20].

Table 2. - Assessment of the need for hospitalization according to the German guidelines for the management of patients with community-acquired pneumonia

Premorbid epidemiological and historical factors

Age $>65$ yrs

Unstable home situation

Comorbidity

COPD or other structural lung disease

Cardiac disease

Renal disease

Hepatic disease

Diabetes mellitus

Alcohol abuse

Malnutrition

Immunosuppression (e.g. by corticosteroids)

Physical findings

Respiratory rate $>30 \cdot \mathrm{min}^{-1}$

Systolic blood pressure $<90 \mathrm{mmHg}$, diastolic blood pressure $<60 \mathrm{mmHg}$

Mental confusion or decreased level of consciousness

Extrapulmonary involvement (septic arthritis, meningitis, etc.)

Radiographic findings

Multilobar and/or bilateral infiltrates

Lobar infiltrates or cavities

Pleural effusions

Laboratory findings

Leukocytosis $>30 \times 10^{9} \cdot \mathrm{L}^{-1}$ or leukopenia $<4 \times 10^{9} \cdot \mathrm{L}^{-1}$

$\mathrm{Pa}, \mathrm{O}_{2}<60 \mathrm{mmHg}$ or $\mathrm{Sa}_{2} \mathrm{CO}_{2}<90 \%$ at $F \mathrm{I}, \mathrm{O}_{2} 0.21$

Parameters indicating renal failure

COPD: chronic obstructive pulmonary disease; $\mathrm{Pa}_{\mathrm{a}} \mathrm{O}_{2}$ : oxygen tension in arterial blood; $\mathrm{Sa}_{\mathrm{a}} \mathrm{CO}_{2}$ : arterial oxygen saturation; $\mathrm{FI}_{\mathrm{I}} \mathrm{O}_{2}$ : inspiratory oxygen fraction. Adapted from [21]. According to these guidelines, hospitalization should be seriously considered in the presence of at least $\geq 2$ adverse premorbid epidemiological and historical factors or one adverse physical finding (or, if available, one radiographic or laboratory finding).
In view of these findings, the German guidelines for the management of CAP have proposed an assessment of the need for hospitalization based on those factors in the list of ATS criteria available after the first clinical evaluation without any further laboratory or radiographic evaluation (table 2) [21]. On the other hand, the ERS guidelines have proposed to consider hospitalization in the presence of: 1) risk factors for severity, 2) risk factors for particular

Table 3. - Criteria for hospitalization according to the European Respiratory Society guidelines

\section{Consideration of \\ hospitalization}

Risk factors for severity

Age $>65$ yrs

Institutionalized patients

Alcoholism

Comorbidity

COPD

Cardiovascular disease

Neurological diseases

Diabetes mellitus

Chronic liver or renal failure

Recent viral infection

Hospital admission within

the previous year

Hospital admission within the previous 2-4 weeks

Recent treatment with penicillin or other antibiotics

Aspiration

\section{Recommendation of} hospitalization

Signs of immediate severity

Home consultation

Chest pain

Confusion

Drowsiness

Tachycardia $\geq 125 \mathrm{bpm}$

Temperature $<35^{\circ} \mathrm{C}$ or

$\geq 40^{\circ} \mathrm{C}$

Respiratory rate $\geq 30 \cdot \mathrm{min}^{-1}$

Cyanosis

Blood pressure $<90 / 60$

$\mathrm{mmHg}$

Complications

Suspected pleural effusion

or cavitation

Metastatic infection

Home management

apparently impossible

Vomiting

Social exclusion

Extreme poverty

Dependency

Poor likelihood of good

compliance

Altered mental status

COPD: chronic obstructive pulmonary disease; GNEB: Gramnegative enteric bacilli.
Risk factors for particular micro-organisms Streptococcus pneumoniae Streptococcus pneumoniae, GNEB, Staphylococcus aureus, anaerobes GNEB, Legionella spp. Streptococcus pneumoniae, Haemophilus influenzae, GNEB

Streptococcus pneumoniae (especially penicillin-resistant strains in some areas)

GNEB

Streptococcus pneumoniae (especially penicillin-resistant strains in some areas), other resistant micro-organisms

GNEB, Staphylococcus aureus, anaerobes 
micro-organisms typically associated with more severe pneumonia, (as defined in table 2, respectively), and 3) failure of first-line antimicrobial treatment [2]. Furthermore, hospitalization is clearly recommended in the presence of signs of immediate severity, complications, and other factors which render home management impossible. As is evident from table 3, most risk factors of severity refer to epidemiological characteristics and comorbid conditions. However, the link of these risk factors for severity and the presence of distinct micro-organisms seems debatable. For example whether CAP in nursing home residents is associated with a distinct microbial pattern is a subject of controversy [6], and whether ambulatory treatment failures are due to resistant microorganisms in a significant proportion of the cases has not been determined.

\section{Predicting death from pneumonia}

Prognostic factors associated with death from pneumonia have been continuously studied in diverse patient populations, and, as outlined in detail in recent reviews covering this subject, more than 40 corresponding predictors in multivariate analyses could be identified $[6,19]$. The adverse independent prognostic factors reported in the last decade are listed in table 4 [22-36]. A metaanalysis comprising of 122 studies dealing with the investigation of prognostic factors found ten independent predictors of death, including male gender, diabetes mellitus, neoplastic disease, neurologic disease, tachypnoea, hypotension, hypothermia, leucopenia, bacteraemia, and multilobar infiltrates, as well as pleuritic chest pain as a protective factor [37].

From a clinical point of view, the authors advocate that it seems useful to arrange these variables similar to the acute physiology and chronic health evaluation (APACHE) score into factors reflecting acute pneumonia relatedillness and those reflecting the underlying health state $[6$, 19]. The former would be further divided into clinical signs and symptoms, laboratory, radiographic, microbiological and oxygenation parameters, whereas the later would include age, sex, referral (home or nursing-home), comorbidity and steroid pretreatment. A third group of parameters would represent evolutionary parameters reflecting disease progression. These factors differ in that they are not available at initial assessment but indicate prognosis during the course of disease. Again, these factors would be divided into clinical, radiographic, and treatment-associated parameters as well as other complications.

If the variety of factors found to be associated with death are examined, it appears that the main general denominators of prognosis include age, male sex, comorbidity, acute respiratory failure, severe sepsis and septic shock, extension of radiographic infiltrates, bacteraemia and CAP through several different pathogens such as $S$. pneumoniae, $S$. aureus, GNEB, and signs of disease progression within the first $48-72 \mathrm{~h}$.

\section{Risk score assessment}

FINE et al. [38] have elaborated risk scores for patients with CAP. In a previous report, a pneumonia (disease-
Table 4. - Independent prognostic factors associated with death from community-acquired pneumonia in studies originating from the last decade including both the general and the intensive care unit treated populations

\begin{tabular}{lc}
\hline Population & Reference \\
\hline General & \\
Respiratory rate $\geq 30 \cdot \mathrm{min}^{-1}$ & $24-26$ \\
Systolic blood pressure $\leq 80 \mathrm{mmHg}$ or & $24-26,28$ \\
$<90 \mathrm{mmHg}$ & \\
Diastolic blood pressure $<60 \mathrm{mmHg}$ & $24-26$ \\
Blood urea nitrogen $>7 \mathrm{mmol} \cdot \mathrm{L}^{-1}$ & $24-26$ \\
Heart rate $\geq 90 \mathrm{bpm}$ & 28 \\
Mental confusion & $24-26$ \\
Low lymphocyte count & 23 \\
Low serum albumin & 23 \\
LDH $\geq 260$ U. ${ }^{-1}$ & 28 \\
Bilateral pleural effusions & 29 \\
Elderly & \\
Temperature $\leq 37^{\circ} \mathrm{C}$ & 30 \\
Respiratory rate $\geq 30 \cdot \mathrm{min}^{-1}$ & 30 \\
Number of affected lobes $\geq 3$ & 30 \\
Bedridden state & 30 \\
ICU-treated general & \\
Age & 33,36 \\
Anticipated death within $4-5$ yrs & 33,36 \\
SAPS >12 or $>13$ & 32,33 \\
Bilateral infiltrates & 33 \\
Requirement for mechanical ventilation & 33,36 \\
Septic shock & $31-34,36$ \\
Involvement $>1$ lobe & 36 \\
Rapid radiographic spread & 31 \\
Inadequate or ineffective intial antimicrobial & 31,34 \\
treatment & \\
Nonpneumonia related complications & 34 \\
Nonaspiration pneumonia & 36 \\
Bacteraemia & 34 \\
Streptococcus pneumoniae & 32 \\
Gram-negative enteric bacilli & 32 \\
Pseudomonas aeruginosa & 33 \\
ICU-treated elderly & \\
Septic shock & 35 \\
Acute renal failure & 35 \\
Rapid radiographic spread & 35 \\
\hline \\
LDHi
\end{tabular}

LDH: lactate dehydrogenase; SAPS: Simplified Acute Physiology Score; ICU: intensive care unit.

specific) prognostic index was derived from around 350 hospitalized patients and validated in more than 14,000 patients of the Medisgroups Comparative Hospital Database. This index included six predictors with an integer value directly proportional to the magnitude of its coefficient in a multivariate mortality model: 1) age $>65$ yrs +1 point, 2) pleuritic chest pain -2 points, 3) vital sign abnormality +2 points, 4 ) altered mental status +2 points, 5) high-risk aetiology +2 points, and 6) neoplastic disease +4 points. Patients were categorized into five risk classes. The index performed particularly well in classifying lowrisk patients (classes I and II). The strength of this prognostic index is clearly its relative simplicity. It can successfully be applicated e.g. in studies comparing different treatment settings in nursing home residents with pneumonia [39]. Conversely, again the inclusion of aetiologic information precludes utilization of this score for clinical decision making within the initial evaluation of the patient. 
In a subsequent study comprising a derivation and validation population of $>50,000$ patients together derived from the Medisgroups and Port cohorts, a two-step risk score was developed [40]. In a first step, the patient with a very low mortality risk (risk class 1 ) is identified by age $<50 \mathrm{yrs}$, lack of comorbidity, and the absence of vital sign abnormalities. In a second step, risk classes II-V are calculated summing up points assigned to age, comorbid conditions, as well as vital sign abnormalities, and diverse epidemiological, laboratory, oxygenation and radiographic features recorded within the first $48 \mathrm{~h}$ after the primary clinical evaluation (table 5). It is noteworthy, that in this risk score age determines the risk class assignment to the largest extent. Additional factors with an exceptionally high impact on the risk score (30 points) include neoplastic disease and mild acidosis.

The first two risk classes were associated with a very low risk of mortality of $<1 \%$, whereas risk classes III-V were associated with a 2.8, 8.2, and 29.2 and 1.2, 9.0, and $27.1 \%$ mortality in the derivation and validation cohorts, respectively. The authors recently validated this rule in an elderly population with CAP. Mortality rates for risk classes II-V (risk class I was absent by definition) were $0,2.7,7.5$, and 30.3\%, respectively [41]. Thus, Fine's classification proved to provide excellent predictions of risk also in this elderly European population.

Fine's study was primarily interested in identifying the patient at low risk who might be safely treated on an outpatient basis. The most useful clue for the practitioner is

Table 5. - Criteria used in the severity assessment model for community-acquired pneumonia (risk classes II-V)

\begin{tabular}{|c|c|}
\hline Criterion & Points \\
\hline Age & Age (yrs) \\
\hline Females & -10 \\
\hline Nursing home residency & 10 \\
\hline \multicolumn{2}{|l|}{ Comorbidity } \\
\hline Neoplastic & 30 \\
\hline Liver & 20 \\
\hline Congestive heart failure & 10 \\
\hline Cerebrovascular disease & 10 \\
\hline Renal disease & 10 \\
\hline \multicolumn{2}{|l|}{ Vital sign abnormality } \\
\hline Mental confusion & 20 \\
\hline Respiratory rate $\geq 30 \cdot \mathrm{min}^{-1}$ & 20 \\
\hline Systolic blood pressure $<90 \mathrm{mmHg}$ & 20 \\
\hline Temperature $<35$ or $\geq 40^{\circ} \mathrm{C}$ & 15 \\
\hline Tachycardia $\geq 125 \mathrm{bpm}$ & 10 \\
\hline \multicolumn{2}{|l|}{ Laboratory abnormalities } \\
\hline Blood urea nitrogen $\geq 11 \mathrm{mmol} \cdot \mathrm{L}^{-1}$ & 20 \\
\hline Sodium $<130 \mathrm{mmol} \cdot \mathrm{L}^{-1}$ & 20 \\
\hline Glucose $\geq 250 \mathrm{mg} \cdot \mathrm{dL}^{-1}$ & 10 \\
\hline Haematocrit <30\% & 10 \\
\hline \multicolumn{2}{|l|}{ Radiographic abnormalities } \\
\hline Pleural effusion & 10 \\
\hline \multicolumn{2}{|l|}{ Oxygenation parameter } \\
\hline Arterial $\mathrm{pH}<7.35$ & 30 \\
\hline$P \mathrm{a}, \mathrm{O}_{2}<60 \mathrm{mmHg}$ & 10 \\
\hline $\mathrm{Sa}_{\mathrm{a}, \mathrm{O}_{2}}<90 \%$ & 10 \\
\hline
\end{tabular}

$\mathrm{Pa}_{\mathrm{a}} \mathrm{O}_{2}$ : oxygen tension in arterial blood; $\mathrm{Sa}_{\mathrm{a}} \mathrm{O}_{2}$ : arterial oxygen saturation. Point scoring system: risk class I: age $<50$, no comorbidity, no vital-sign abnormality; risk class II: $\leq 70$ points; risk class III: 71-90 points; risk class IV: 91-130 points; risk class V: $>130$ points. the fact that a patient with risk class I has a risk of mortality of $<1 \%$ and therefore, is the ideal candidate for ambulatory treatment. Although patients with risk classes II and III also have a very low risk of death from CAP, the assignment into these classes requires a tedious calculation and this might not be suitable in routine practice.

Conversely, this classification may prove very useful in further studies assessing processes of care and medical outcomes. Accordingly, the authors recently evaluated the management of patients with CAP admitted to a primary care hospital. It was found that principal conceptual weaknesses in this particular setting which might be subject to intervention were: 1) the hospitalization of patients with mild pneumonia at low risk of mortality; 2) a lack of association between microbial investigation and severity of CAP; 3 ) antimicrobial overtreatment of patients with nonsevere CAP; 4) inadequate antimicrobial treatment particularly in patients with severe CAP; and 5) an increased number of primary treatment failures and duration of hospitalization [42].

When using this risk score in routine practice, a note of caution should be made. Although mortality rates in risk classes I-III do not exceed 3\%, they are not zero. Accordingly, single patients initially assigned to risk class I and II may even eventually require ICU admission [40, 41]. Thus, it is important to realize that patients with CAP and also at low risk of death should be re-examined clinically for possible unexpected deterioration. In this regard, a recent study provided very useful information about the time course of stability after the initiation of initial empiric antimicrobial treatment [43]. In hospitalized patients, the median time to overall clinical stability was 3 days for the most lenient definition of stability and 7 days for the most conservative definition. Time to stability was clearly correlated to initial severity. Once stability was achieved, clinical deterioration requiring ICU treatment occurred in $<1 \%$ of cases. Similarly, in the recent series of severe CAP it was found that $80 \%$ of patients requiring ICU treatment were admitted within $24 \mathrm{~h}$, and an additional 15\% within $72 \mathrm{~h}$ [44]. Overall, these findings indicate that in patients with initially mild CAP treated on an outpatient basis, most deteriorating courses will occur within the first $24-72 \mathrm{~h}$ after the initiation of empiric antimicrobial treatment. Thus, any ambulatory treatment should prompt a reassessment of pneumonia severity and the clinical course within this time period.

Only recently, another interesting prediction rule for mortality from severe CAP treated in ICUs has been proposed [36]. In this study of 472 eligible patients with severe CAP, the following six variables available at initial evaluation were independently associated with death: 1) age $\geq 40 \mathrm{yrs}, 2$ ) anticipated death within 5 yrs, 3) nonaspiration pneumonia, 4) chest radiograph involvement $>1$ lobe, 5) acute respiratory failure requiring mechanical ventilation, and 6) septic shock. Based on these factors, each factor was assigned a point value, and all the factors had a point value of 1 except septic shock which had a point value of 3 . The resulting risk score included three classes of increasing mortality. The main clue from this study was that whereas low-risk (point score 0-2) and high-risk (point score 6-8) patients could be confidently identified, the outcome of intermediate-risk patients was not predictable unless adjusted for evolutionary 
factors independently associated with death in an additional analysis accounting for these factors only. The impact of three factors (hospital-acquired lower respiratory tract superinfections, nonspecific CAP-related complications, and sepsis-related complications) on the outcome prediction was dramatic, indicating that it is largely determined by (initially hardly predictable) complications which occur after ICU admission.

\section{Prognostic rules for the individual hospitalized patient}

Since the report by the British Thoracic Society (BTS) on CAP, indicating that inhospital outcome of the individual patient can accurately be predicted by prognostic rules including three simple clinical or laboratory parameters, there has been considerable interest in validating these rules [22, 24-27, 45, 46] (table 6). Several studies could confirm excellent operative characteristics of the original $[24,25]$ or a slightly modified rule [26]. The sensitivities ranged from 70-90\%, and the specificities from $76-84 \%$ (table 6). More recently, two studies have failed to confirm these favourable prediction results, one

Table 6. - Prognostic rules for the individual outcome in patients with community-acquired pneumonia and their performances

\begin{tabular}{|c|c|c|c|c|}
\hline & Sens. & Spec. & PPV & NPV \\
\hline \multicolumn{5}{|l|}{ Rule 1} \\
\hline Derivation study [22] & 88 & 79 & 19 & 99 \\
\hline \multicolumn{5}{|l|}{ Validation studies } \\
\hline FARR et al. [24] & 70 & 84 & 29 & 97 \\
\hline KaRALUS et al. [25]* & 83 & 80 & 23 & 99 \\
\hline NeILl et al. [26] & 90 & 76 & 25 & 99 \\
\hline Lim et al. [27] & 52 & 79 & NR & NR \\
\hline EWIG et al. [41]** & 65 & 73 & 21 & 95 \\
\hline CONTE et al. $[45]^{* *}$ & 50 & 70 & NR & NR \\
\hline \multicolumn{5}{|l|}{ Rule 2} \\
\hline Derivation study [22] & 39 & 94 & 36 & 97 \\
\hline \multicolumn{5}{|l|}{ Validation studies } \\
\hline FARR et al. [24] & 35 & 89 & 22 & 94 \\
\hline NeILL et al. [26] & 65 & 88 & 33 & 97 \\
\hline EWIG et al. [41]** & 47 & 88 & 31 & 94 \\
\hline \multicolumn{5}{|l|}{ Rule 3} \\
\hline Derivation study [26] & 95 & 71 & 22 & 99 \\
\hline Validation study [27] & 66 & 73 & NR & NR \\
\hline \multicolumn{5}{|l|}{ Rule 4} \\
\hline Derivation study [28] & 77 & 75 & 42 & 93 \\
\hline Validation study $[41]^{* *}$ & 47 & 80 & 21 & 93 \\
\hline
\end{tabular}

Sens.: sensitivity; Spec.: specificity. PPV: positive predictive value; NPV: negative predictive value; NR: not reported. Rule 1: (original BTS-rule 1): at least two of three of the following: respiratory rate $\geq 30 \cdot \mathrm{min}^{-1}$, diastolic blood pressure $\leq 60$ $\mathrm{mmHg}$, blood urea nitrogen $>7 \mathrm{mmol} \cdot \mathrm{L}^{-1}$; Rule 2: (original BTS-rule 2): at least two of three of the following: respiratory rate $\geq 30 \cdot \mathrm{min}^{-1}$, diastolic blood pressure $\leq 60 \mathrm{mmHg}$, mental confusion; Rule 3: (modified BTS-rule): at least two of four of the following: respiratory rate $\geq 30 \cdot \mathrm{min}^{-1}$, diastolic blood pressure $\leq 60 \mathrm{mmHg}$, blood urea nitrogen $>7 \mathrm{mmol} \cdot \mathrm{L}^{-1}$, mental confusion; Rule 4: (EwIG et al.): at least two of three of the following: systolic blood pressure $\leq 80 \mathrm{mmHg}$, heart rate $\geq 90$ $\mathrm{bpm}, \mathrm{LDH} \geq 260 \mathrm{U} \cdot \mathrm{L}^{-1}$. $*$ : using rule 1 or rule $2 ; * *$ : elderly population $(\geq 65 \mathrm{yrs})$. in a general [27] and another in an elderly population [41].

Since the majority of patients with CAP is expected to belong to the elderly population, the authors were particularly interested in the performance of the original BTS rules in these elderly patients. It was hypothesized that elevated blood urea nitrogen which is more frequently present or at least can more readily develop in the elderly would represent a confounding factor whereas mental confusion as a marker of severe sepsis would work particularly well in this frequently oligosymptomatic population. In fact, it was found that blood urea nitrogen only had a very low specificity whereas the opposite was true for mental confusion. Accordingly, the second BTS rule had the best performance as evidenced by operative indices (sensitivity $47 \%$, specificity $88 \%$ ) as well as by risk assignment of prognostic rules according to risk classes as proposed by Fine et al. [40].

With regards an alternative rule proposed by the authors [28], a satisfactory specificity of $80 \%$ could be confirmed but only a very limited sensitivity of $47 \%$ in this elderly population was found [41].

Looking at the performances of these rules, two issues should be pointed out. First, the predictive power of these simple rules is considerably high. This can best be explained if it is considered that these rules include parameters mainly reflecting two of the most important prognostic factors, i.e. acute respiratory failure (by respiratory rate $\geq 30 \cdot \mathrm{min}^{-1}$ ) and severe sepsis or septic shock (by blood urea nitrogen, mental confusion, hypotension, or tachycardia). Secondly, since specificity was consistently found to be high but sensitivity to be quite variable, the true strength of these rules is their negative predictive value, i.e. the identification of patients who are not at risk of death from pneumonia. This value was found to exceed $90 \%$ in all validation studies published so far [24-27, 41]. Nevertheless, since these rules were derived and validated only in hospitalized patients, the applicability of these rules in triaging outpatients for hospitalization or ICU admission is not settled.

\section{Defining severe pneumonia}

Since mortality from CAP is consistently highest in patients who require admission at the ICU, ranging 25$50 \%[6,19]$, it appears particularly important to identify such patients at highest risk for death in order to ensure intensive care as early as possible. Although since 1985 $>30$ series of severe CAP have been published, the only common denominator of pneumonia severity in these studies was the decision to admit the patient at the ICU in the particular hospital. Therefore, it was thought that one important way to identify objective severity criteria behind these decisions would be to determine the predictive power of such criteria for the decision to refer the patient to the ICU in an experienced tertiary care centre.

The severity criteria as suggested by the ATS guidelines for the management of adult CAP was primarily evaluated $[1,44]$ (table 7). With the exception of the oxygenation index, all nine remaining severity criteria were significantly associated with death. Likewise, except respiratory rate $>30 \cdot \mathrm{min}^{-1}$ and the oxygenation index, specificity of the remaining eight severity criteria was high, ranging 
Table 7. - Criteria for the definition of severe communityacquired pneumonia as suggested by the American Thoracic Society (ATS)

Baseline ("minor") criteria assessed at admission

1. Respiratory rate $>30 \cdot \mathrm{min}^{-1}$

2. Severe respiratory failure $\left(\mathrm{Pa}, \mathrm{O}_{2} / F \mathrm{I}, \mathrm{O}_{2}<250\right)$

3. Bilateral involvement in chest radiograph

4. Involvement of $>2$ lobes in chest radiograph (multilobar involvement)

5. Systolic blood pressure $<90 \mathrm{mmHg}$

6. Diastolic blood pressure $<60 \mathrm{mmHg}$

"Major" criteria assessed at admission or during clinical course

1. Requirement for mechanical ventilation

2. Increase in the size of infiltrates by $\geq 50 \%$ in the presence of clinical nonresponse to treatment or deterioration (progressive infiltrates)

3. Requirement of vasopressors $>4 \mathrm{~h}$ (septic shock)

4. Serum-creatinine $\geq 2 \mathrm{mg} \cdot \mathrm{dL}^{-1}$ or increase of $\geq 2$ $\mathrm{mg} \cdot \mathrm{dL}^{-1}$ in a patient with previous renal disease or acute renal failure requiring dialysis (acute renal failure)

Modified severity criteria as suggested by EwIG et al. [44] include: at least two of three of the baseline ("minor") criteria 2, 4, and 5; or at least one of two "major" criteria 1 and 3. Data as suggested by the ATS [1].

$86-100 \%$, but at the cost of a low sensitivity (range $12-$ $52 \%$ ). Similar performances were found for additional severity criteria such as mental confusion, tachycardia $>125 \mathrm{bpm}$, hypercapnia and the presence of pleural effusion. However, when the recommendation of the ATS guidelines to consider ICU admission in the presence of at least one criterion out of the 10 severity criteria was applied, sensitivity was $98 \%$ but specificity only $32 \%$. Thus, this rule would clearly result in oversensitivity and be a poor predictor of the patient at risk.

In order to develop a more balanced predictive rule of pneumonia severity, it was thought that it would be important to rearrange the ATS severity criteria according to those available after initial clinical examination (baseline parameters) and those which are assessed either at admission or during clinical course and clearly imply more severe illness (major criteria). Within both groups of parameters, those independently associated with severity were assessed by multivariate analysis, and these parameters were tested for their ability to predict pneumonia severity (table 7). The presence of two of three baseline criteria had a sensitivity of $33 \%$ and a specificity of $94 \%$. However, if severe CAP was defined as the presence of two of three baseline or one of two major criteria, the performance was more balanced, with a sensitivity of $75 \%$, a specificity of $94 \%$, and a positive predictive value of $74 \%$ and a negative predictive value of $95 \%$. Thus, it appears that those five parameters should be able to identify the patient with severe CAP quite accurately. However, this rule is pending a prospective validation in an independent patient cohort. Moreover, in addition to this rule, the clinician should remain alert to other criteria of severity, such as the presence of mental confusion and pleural effusion.

One of the most striking findings in this study was the low predictive power of parameters reflecting acute respiratory failure. However, both the respiratory rate and the oxygenation index may result in greater accuracy after the application of a defined amount of oxygen for a defined amount of supplemental oxygen, thus correcting for biases arising from the stress of the prehospitalization period.

A clear limitation of the suggested definition lies in the mix-up of baseline and potentially evolutionary criteria. An important minority of patients who do not meet severity criteria on admission may nevertheless be at high risk of developing severe CAP in the following $72 \mathrm{~h}$ but not receive intensive care and corresponding appropriate antimicrobial treatment. Therefore, predictors of a high risk for clinical deterioration requiring ICU treatment need to be developed which allow for the appropriate assessment of the severity of pneumonia in these patients also.

\section{Severity assessment as part of new concepts of patient management}

The assessment of severity of CAP is not only crucial for decisions about hospitalization and ICU admission but also for the selection of initial antimicrobial treatment. As outlined in the introduction, the classification into mild, moderate, and severe CAP is usually synonymous with the estimation that, as far as pneumonia severity is concerned, the patient can be treated as an outpatient or must be hospitalized or admitted to the ICU, respectively. Moreover, distinct empiric antimicrobial regimen are being recommended according to these three categories of severity based on estimations about microbial patterns associated with these categories [1-6].

More recently, new concepts have been developed which include the assessment of severity into decisions about the intensity of antimicrobial treatment and length of hospital stay. In one retrospective study, patients were classified as being low risk in case of absence of: 1) obvious reasons for continued hospitalization on the third hospital day, 2) a high-risk aetiology, and 3) a lifethreatening complication within the first 3 days of hospitalization. Of 503 eligible hospitalized patients, 33\% were classified as being at low risk according to these criteria and potentially suitable for early conversion to oral antimicrobial treatment [47]. In another concept, low risk patients as defined by the absence of risk factors associated with pneumonia-related morbidity or mortality are treated as outpatients with oral antibiotics whereas high risk patients are hospitalized and initially treated with intravenous antibiotics. This latter group is further subdivided into: 1) patients with primarily unstable pneumonia as defined by the presence of severe underlying comorbidity and other adverse prognostic factors; these patients are closely observed and rapidly switched to oral antibiotics and discharged in case of reaching clinical stability; and 2) patients with primarily unstable pneumonia who deteriorate and experience complicated pneumonia as defined by advanced local disease, metastatic spread of infection or systemic decompensation; these patients receive prolonged intravenous antibiotic treatment [48].

These concepts are particularly attractive because they provide a framework for the study of the optimal duration, the selection of routes and the accurate time-point to switch from intravenous to oral antimicrobial treatment in different groups at risk. Likewise, together with data provided by aforementioned studies assessing the time to 
clinical stability, they may be of help in optimizing the application of supportive treatment measures and duration of hospitalization. Results of these studies are likely to change the conventional management attitudes and thereby, contribute to a substantial cost-saving in the treatment of patients with CAP.

\section{Conclusions and future prospects}

The assessment of severity plays a crucial role in the management of patients with CAP. This is true for the evaluation of the patient with LRTI, the triaging decision about hospitalization or ICU admission, and, as a consequence, selection of initial antimicrobial treatment. Severity assessment has also been increasingly applied in studies evaluating the optimal selection of routes of antimicrobial treatment application, duration of antimicrobial treatment and hospital discharge.

Factors clearly associated with adverse outcomes have been incorporated in the recommendations about severity assessment of the current guidelines for the management of adult CAP. However, beyond some clear-cut severity criteria some important topics of severity assessment remain doubtful. This is particularly true for the evaluation of patients with mild LRTI and the definition of severe CAP requiring ICU admission. Future investigations should determine whether the confirmation of infiltrates in chest radiographs of patients with mild LRTI and suspected CAP has prognostic weight important enough to render regular chest radiographs a mandatory goal of severity assessment. When attempting to improve the definitions of severe CAP, it will be important to identify predictors of imminent deterioration within the $72 \mathrm{~h}$ following initial empiric antimicrobial treatment.

Currently available tools for the assessment of severity of the individual patient such as prediction rules for ambulatory patients at risk for complications without obvious reasons for hospitalization [17] and for death from pneumonia in hospitalized patients [22, 24-28, 41] are simple enough to be clinically applicable. The identification of objective criteria for severe pneumonia requiring ICU admission may allow more accurate allocation of limited health care resources. Further studies will be necessary to validate and refine these or modified predictive rules in different settings. However, all predictive rules will remain far from being perfect, and the appropriate estimation of the individual risk and medical as well as nonmedical requirements will always remain a medical art.

On the other hand, risk classifications such as the scores developed by FINE et al. [40] are not only useful in identifying the patients at low risk who might safely be treated as outpatients but will play a major role in the evaluation of processes and outcomes of care for patients with CAP. Based on such evaluations it will be possible to define interventions aimed at reducing hospital admission rates, defining risk-adapted antimicrobial treatment regimen, reducing costs for antimicrobial treatment and supportive measures, and shortening of hospital stay. Together with these standardizations, it will be possible to recognize the true peculiarities of the particular treatment setting and to account for them appropriately. This will most likely contribute to an increased acceptance of management guidelines throughout different settings. Overall, it is to be expected that the quality of care can be substantially improved by these tools of severity assessment, and further corresponding work is anticipated in the near future.

\section{References}

1. American Thoracic Society. Guidelines for the initial management of adults with community-acquired pneumonia: diagnosis, assessment of severity, and initial antimicrobial therapy. Am Rev Respir Dis 1993; 148: 1418-1426.

2. European Study on Community-acquired pneumonia (ESOCAP) Commitee. Guidelines for management of adult community-acquired lower respiratory tract infections. Eur Respir J 1998; 11: 986-991.

3. Huchon G, Woodhead M. Management of adult community-acquired lower respiratory tract infections. Eur Respir Rev 1998; 8: 391-426.

4. Guidelines from the Infectious Diseases Society of America. Community-acquired pneumonia in adults: guidelines for management. Clin Infect Dis 1998; 26: 811-838.

5. Ruiz, M, Ewig S, Marcos MA, et al. Etiology of community-acquired pneumonia - impact of age, comorbidity, and severity. Am J Respir Crit Care Med 1999; 160: 397405.

6. Ewig S. Community-acquired pneumonia. Epidemiology, risk, and prognosis. Eur Respir Mon 1996; 3: 13-35.

7. Woodhead MA, Macfarlane JT, McCracken JS, Rose DH, Finch RG. Prospective study of the etiology and outcome of pneumonia in the community. Lancet 1987; 1: 671674.

8. Diehr P, Wood RW, Bushyhead J, Krueger L, Wolcott B, Tompkins RK. Prediction of pneumonia in outpatients with acute cough: a statistical approach. J Chronic Dis 1984; 37: 215-225.

9. Gennis P, Gallagher J, Falvo C, Baker S, Than W. Clinical criteria for the detection of pneumonia in adults: guidelines for ordering chest roentgenograms in the emergency department. J Emerg Med 1989; 7: 263-268.

10. Singal BM, Hedges JR, Radack KL. Decision rules and clinical prediction of pneumonia: evaluation of low-yield criteria. Ann Emerg Med 1989; 18: 13-20.

11. Heckerling PS, Tape TG, Wigton RS, et al. Clinical prediction rule for pulmonary infiltrates. Ann Intern Med 1990; 113: 664-670.

12. Melbye H, Dale K. Interobserver variability in the radiographic diagnosis of adult outpatient pneumonia. Acta Radiol 1992; 33: 79-81.

13. Young $M$, Marrie TJ. Interobserver variability in the interpretation of chest roentgenograms of patients with positive pneumonia. Arch Intern Med 1994; 154: 2729 2732.

14. Macfarlane JT, Colville A, Gulon A, Mcfarlane RM, Rose DH. Prospective study of aetiology of adult lower respiratory tract infections in the community. Lancet 1993; 341: 511-514.

15. Emerman CL, Dawson N, Speroff T, et al. Comparison of physician judgment and decision aids for ordering chest radiographs for pneumonia in outpatients. Ann Emerg Med 1991; 20: 1215-1219.

16. Macfarlane J. Lower respiratory tract infection and pneumonia in the community. Semin Respir Infect 1999; 14: 151-162. 
17. Black ER, Mushlin AI, Griner PF, Suchman AL, James RL, Schoch DR. Predicting the need for hospitalization of ambulatory patients with pneumonia. J Gen Intern Med 1991; 6: 394-400.

18. Fine MJ, Smith DN, Unger DE. Hospitalization decision in patients with community-acquired pneumonia: a prospective cohort study. Am J Med 1990; 89: 713-721.

19. Ewig S. Community-acquired pneumonia: definition, epidemiology, and outcome. Semin Respir Infect 1999; 14: 94-102.

20. Gordon G, Throop D, Berberian L, Niederman MS, Bass $\mathrm{J}$ Jr, Alemayehu D. Validation of the American Thoracic Society Guidelines for community-acquired pneumonia in hospitalized patients. Am J Respir Crit Care Med 1996; 153: A257.

21. Schaberg T, Dalhoff K, Ewig S, Lorenz J, Wilkens H. Deutsche Gesellschaft für Pneumologie. Empfehlungen zur Diagnostik und Therapie der ambulant erworbenen Pneumonie. Pneumologie 1998; 52: 450-462.

22. British Thoracic Society and the Public Health Laboratory Service. Community-acquired pneumonia in adults in British hospitals in 1982-1983: a survey of aetiology, mortality, prognostic factors and outcome. Q J Med 1987; 239: 195-220.

23. Örtquist A, Hedlund J, Grillner L, et al. Aetiology, outcome and prognostic factors in community-acquired pneumonia requiring hospitalization. Eur Respir J 1990; 3: 1105-1113.

24. Farr BM, Sloman AJ, Fisch MJ. Predicting death in patients hospitalized for community acquired pneumonia. Ann Intern Med 1991; 115: 428-436.

25. Karalus NC, Cursons RT, Leng RA, et al. Community acquired pneumonia: aetiology and prognostic index evaluation. Thorax 1991; 46: 413-418.

26. Neill AM, Martin IR, Weir R, et al. Community-acquired pneumonia: aetiology and usefulness of severity criteria on admission. Thorax 1996; 51: 1010-1016.

27. Lim WS, Lewis S, Macfarlane JT. Severity prediction rules in in community-acquired pneumonia: a validation study. Thorax 2000; 55: 219-223.

28. Ewig S, Bauer T, Hasper E, Pizulli L, Kubini R, Lüderitz B. Prognostic analysis and predictive rule for outcome of hospital-treated community-acquired pneumonia. Eur Respir J 1995; 8: 392-397.

29. Hasley PB, Albaum MN, Yi-Hwei L, et al. Do pulmonary radiographic findings at presentation predict mortality in patients with community-acquired pneumonia? Arch Intern Med 1996; 156: 2206-2212.

30. Riquelme R, Torres A, El-Ebiary M, et al. Communityacquired pneumonia in the elderly. A multivariate analysis of risk and prognostic factors. Am J Respir Crit Care Med 1996; 154: 1450-1455.

31. Torres A, Serra-Batiles J, Ferrer A, et al. Severe community acquired pneumonia. Epidemiology and prognosis factors. Am Rev Respir Dis 1991; 114: 312-318.

32. Moine P, Vercken JB, Chevret S, Chastang C, Gajdos P. Severe community-acquired pneumonia. Etiology, epide- miology, and prognosis factors. Chest 1994; 105: $1487-$ 1495.

33. Almirall J, Mesalles E, Klamburg J, Parra O, Agudo A. Prognostic factors of pneumonia requiring admission to the intensive care unit. Chest 1995; 107: 511-516.

34. Leroy O, Santre C, Beuscart C, et al. A five-year study of severe community-acquired pneumonia with emphasis on prognosis in patients admitted to an intensive care unit. Int Care Med 1995; 21: 24-31.

35. Rello J, Rodriguez R, Jubert P, Jubert P, Alvarez B. Severe community-acquired pneumonia in the elderly: epidemiology and prognosis. Clin Infect Dis 1996; 23: 723-728.

36. Leroy O, Devos P, Guery B, et al. Simplified prediction rule for prognosis of patients with severe communityacquired pneumonia in ICUs. Chest 1999; 116: 157-165.

37. Fine MJ, Smith MA, Carson CA, et al. Prognosis and outcome of patients with community-acquired pneumonia: a meta-analysis. JAMA 1996; 275: 134-141.

38. Fine MJ, Singer DE, Hanusa BH, Lave JR, Kapoor WN. Validation of a pneumonia prognostic index using the MedisGroups Comparative Hospital Database. Am J Med 1993; 94: 153-159.

39. Mylotte JM, Naughton B, Saludades C, Maszarovics Z. Validation and application of the pneumonia prognosis index to nursing home residents with pneumonia. $\mathrm{J} \mathrm{Am}$ Geriatr Soc 1998; 46: 1538-1544.

40. Fine MJ, Auble TE, Yealy DM, et al. A prediction rule to identify low-risk patients with community-acquired pneumonia. $N$ Engl J Med 1997; 336: 243-250.

41. Ewig S, Kleinfeld T, Bauer T, Seifert K, Schäfer H, Göke $\mathrm{N}$. Comparative validation of prognostic rules for community acquired pneumonia in an elderly population. Eur Respir J 1999; 14: 370-375.

42. Ewig S, Seifert K, Kleinfeld T, Göke N, Schäfer H. Management of patients with community-acquired pneumonia in a primary care hospital - a critical evaluation. Respir Med 2000; 194: 556-563.

43. Halm EA, Fine MJ, Marrie TJ, et al. Time to clinical stability in patients hospitalized with community-acquired pneumonia: implications for practice guidelines. JAMA 1998; 279: 1452-1457.

44. Ewig S, Ruiz M, Mensa J, et al. Severe communityacquired pneumonia - assessment of severity criteria. Am $J$ Respir Crit Care Med 1998; 158: 1102-1108.

45. Conte Conte HA, Chen YT, Mehal W, Scinto JD, Quagliarello VJ. A prognostic rule for elderly patients admitted with community-acquired pneumonia. $\mathrm{Am} \mathrm{J}$ Med 1999; 106: 20-28.

46. Woodhead M. Predicting death from pneumonia. Thorax 1996; 51: 970-971.

47. Weingarten SR, Riedinger MS, Varis G, et al. Identification of low-risk hospitalized patients with pneumonia. Implications for early conversion to oral antimicrobial therapy. Chest 1994; 105: 1109-1115.

48. Siegel RE. Strategies for early discharge of the hospitalized patient with community-acquired pneumonia. Clin Chest Med 1999; 20: 599-605. 